\title{
Random Word Retrieval for Automatic Story Generation
}

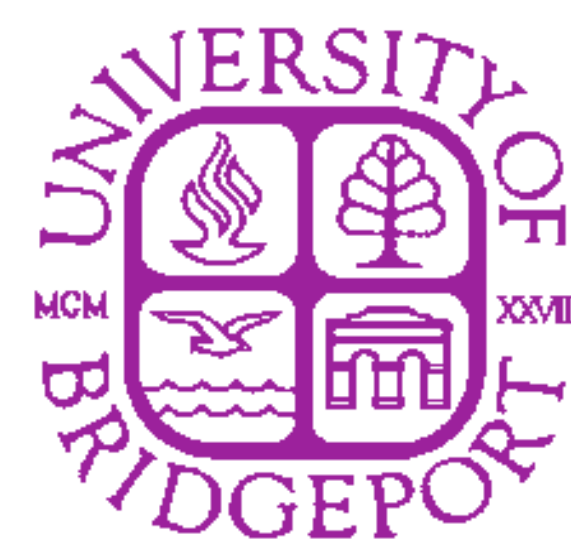

Richard S. Colon Sr. ${ }^{1}$

Prabir K. Patra ${ }^{2,3}$ and Khaled M. Elleithy ${ }^{1}$

'Department of Computer Science and Engineering

2Department of Biomedical Engineering

${ }^{3}$ Department of Mechanical Engineering

University of Bridgeport, Bridgeport, CT

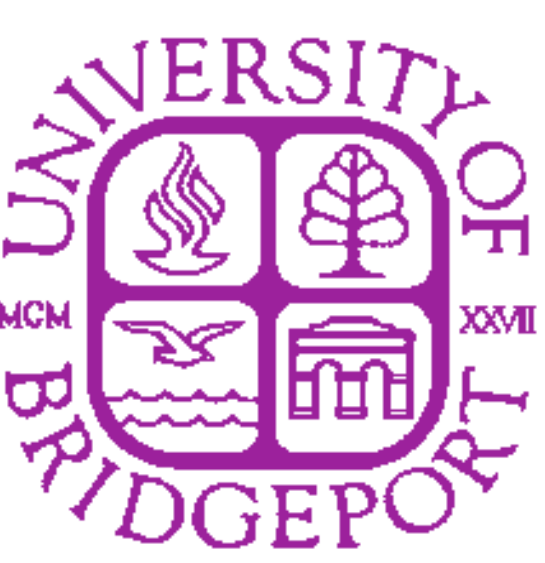

\section{Abstract}

Over the past forty years, significant research has been done on story/narrative generation, in which the computer is the author. While it is impossible to quantify a human writer's inspiration, we can consider a common exercise that authors perform; namely 'writing prompts'. A writing prompt is just a topic or idea around which to start writing. The prompt can simply be a few words, which becomes the basis for a story. Our automatic story generation system, will select a few random words as parameters (i.e. 'inspiration') for driving the 'creative writing' process. Here we present the results generated by the initial phase of system development and testing of the word selection components.

\section{Introduction}

The goal of our story generation system is not to understand how human authors create stories, but to focus solely on the generation of what may be perceived as 'creative' results.

Utilizing the Internet, a few random words will be selected which will form the basic parameters for generating a fictional story. Also, since a machine has no understanding of word concepts within any specific context, we will utilize the Internet (and existing 'Concept Knowledge' systems) to find the context for the selected words, thus guiding the story generation process. The systems used are:

WordNet (Princeton University) - An English lexical database of nouns, adjectives, verbs and adverbs.

ConceptNet (MIT) - A 'commonsense knowledge' database which is used to perform textual-reasoning in order to make sense of the everyday world.

\section{System Design}

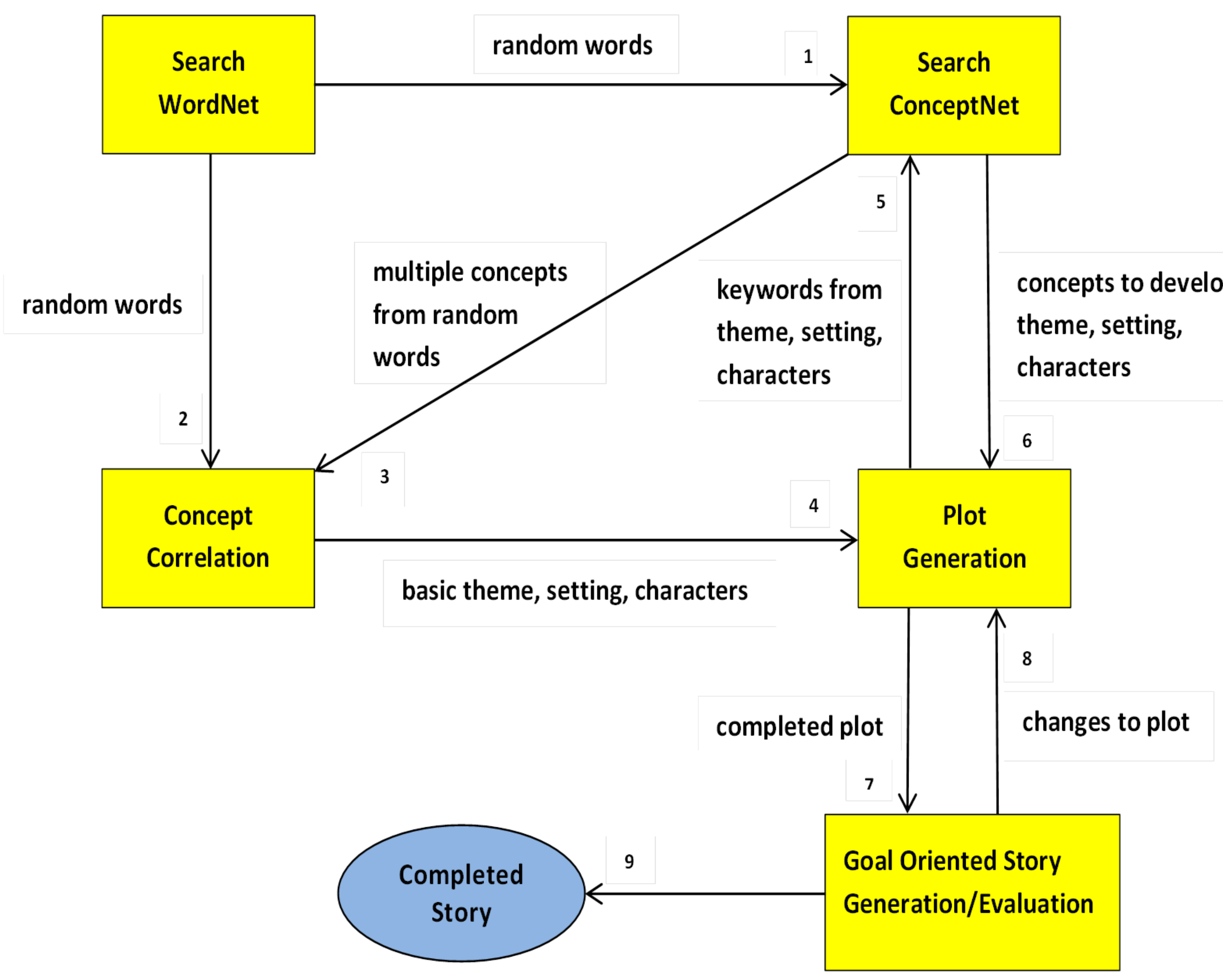

\section{Story Generation Block Diagram}

Following the diagram, the first three flows have been implemented:

1. Via the Internet, use WordNet to retrieve 3 random words. Forward the selected words for concept lookup.

2. Also forward the selected words for Concept Correlation.

3. Via the Internet, use ConceptNet to lookup the conceptual meaning of the selected words. There will usually be multiple different concepts found. Forward all concepts for Concept Correlation.

Written in the Python programming language, our program reads three random words from the WordNet corpus. Using WordNet gives the advantage of selecting words by POS (part of speech) and frequency of use.

For the initial tests, we selected three random words comprised of two nouns and one verb. Since WordNet provides multi-word and hyphenated phrases, these have been filtered out for our tests. Then each of the selected words is used to search ConceptNet.

The results being presented here are sample outputs from the word selection and concept lookup code.

\section{Generated Results}

Python 2.7.5 (default, May 15 2013, 22:43:36) [MSC v.1500 32 bit (Intel)] on win32

$>>>$

Random Word Selection -

Nouns:

Synset('orchestration $n \cdot 02$ ')

Synset('inscription.n.03')

Verb:

Synset('erase.v.03')

(Now the program searches for the concepts associated for each word)

Search word: orchestration

Concept: orchestrate/v/write_an_orchestra_score_for

orchestration/n/the

Concept: orchestration/n/an_arrangement_of_a_piece_of_music_for_performance_by_an_orchestra_or_band

Concept: arrangement/n/the_act_of_arranging_and_adapting_a_piece_of_music

Concept: mastermind $/ \mathrm{v} / \mathrm{plan}$ and_direct

Concept: orchestration/n/an_arrangement_of_events_that_attempts_to_achieve_a_maximum_effect

- ${ }_{\text {Corinstruments }}$

Cor

(NOTE: Most concepts are music related, but it is interesting to see the reference of a "mastermind")

Search word: inscription

Concept: write

Concept: inscription

Concept: epigraph/n/an_engraved_inscription

Concept: inscription/n/letters_inscribed_on_something

Concept: epitaph/n/an_inscription_on_a_tombstone_or_monument_in_memory_of the _person_buried_there

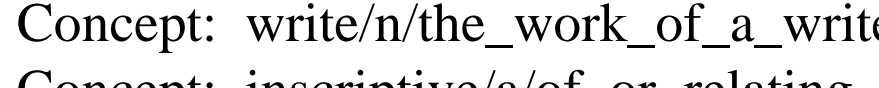

Conet: superscription_n___nding_to_an_inscription

Concept: inscribe/v/write,_engrave,or_print_as_above_something_else

Concept: inscription/n/the activity_of_inscribing _letters_or_word

Concept: superscription/n/the_activity_of_superscribing

Concept: write/n/the_activity_of_putting_something in_written_form

Concept: mark

Concept: legend $/ \mathrm{n}$

Concept. inscription/n/carved_text

Concept. inscription/n/dedication_in_a_book

Concept: inscription/n/text_on_a_coin

Concpt: inscription/n/legend,_writing

Concept: inscriptionally

Concept: inscription_in_wood_or_stone

Concept: inscription_on_tomb

Concept: inscription_of_scripture_on_stone

Concept: inscription_on_tombstone

(NOTE: Most concepts relate to letters and text or writing, yet to have tombstone listed is less ordinary)

Search word: erase

Concept: pen

Wipe_out_digitally_or_magnetically_recorded_information

Concept: take_away/v/take_out_or_remove

Concept: rub/v/move_over_something_with_pressure

Concept: erase/v/remove_by_or_as_if_by_rubbing_or_er

Concept: erase/v/remove_from_memory_or_existence

Concept: expunction/n/deletion_by_an_act_of_expunging_or_erasing

Concept: scratch_out/v/strike_or_cancel_by_or_as_if_by_rubbing_or_crossing_out

Concept: eraser/n/an_implement_used_to_erase_something

Concept: wipe/v/rub_with_a_circular_motion

Concept: demagnetize/v/erase

Concept: erasure/n/a_correction_made_by_erasing

Concept: record/v/register_electronically

Concept: kill/v/cause_to_di

Concept: destruction/n/an_event_that_completely_destroys_something

Concept: sponge/v/erase_with_a_sponge

Concept: erasure/n/a_surface_area_where_something_has_been_erased

Cont: record/nthe_at_of_natisisible

Concept: mistake

Concept: chalk_on black_board

(NOTE: Listed are the different ways and things that can erase or be erased, but kill or "erase a person" was a surprising concept)

End of Program

\section{Conclusion}

The program selected three random words and retrieved a list of concepts. Which of the concepts should be selected? Our selection criteria should be based upon some cohesion between the concepts and the potential for story generation. From a human perspective, we can see the correlation between:

Search word - orchestration: Concept - mastermind, plan_and_direct

Search word - inscription: Concept - inscription_on_tombstone

Search word - erase: Concept - kill, cause_to_die

These concept selections could generate a story about an evil mastermind who plans to kill his mortal enemy and has already inscribed the tombstone. Our goal is for the system to automatically derive a similar (or at least coherent) storyline.

The results generated by the current system implementation are clearly just the beginning. However, this work forms the foundation for development of additional and more complex system components.

References:

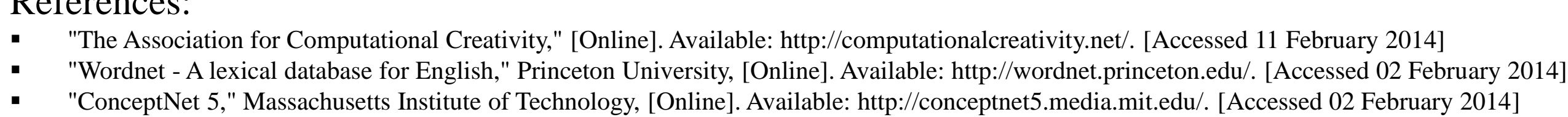

